\title{
Mental Health in Japan: Intersecting Risks in the Workplace
}

\author{
Dave Pilla a and Judy Kurianskya
}

Japanese culture tends to value group harmony and social conformity. The work culture in Japan reflects this belief system, evidenced by employees working significant amounts of overtime, which is often unpaid, and taking minimal vacation. Japan also has a relatively recent history of economic instability, coupled with workers' fears and strong negative perceptions of being fired or unemployed, which creates heightened pressures to fit in as an employee. These pressures, on top of tendencies to overwork, translate into an increased risk of stress and other mental health issues. Furthermore, structural and cultural stigma against mental illness and treatment in Japan acts as a barrier to addressing these mental health risks. In recent years, the Japanese government has stepped up efforts to improve its mental health care system, resulting in some promising developments, particularly pertaining to suicide prevention. However, initiatives to improve mental health at the workplace are still underdeveloped, and lack scope and empirical-basis. For Japanese workers, a framework for targeted improvements can focus on three intersecting mental health risk factors: work culture, financial insecurity, and stigma.

Keywords: Japan; Mental Health; Stigma; Culture; Workplace

\section{Culture and Work in Japanese Society}

The culture of Japan, like many Asian nations, is commonly described as collectivist, in which the group is prioritized over the individual. The importance of maintaining group harmony is considered a defining characteristic of Japanese culture, and while the attribution of this ideology to Japanese people may risk overgeneralization, it does appear to form the basis for some patterns of social behavior observed in Japanese society. The Japanese have been described as tending to conform to social norms and rules, not display emotions in public settings, avoid disagreement, and be sensitive to how they are viewed by others (Davies \& Ikeno, 2002, Russell, Metraux, \& Tohen, 2016). In a study comparing undergraduate student views in individualistic versus collectivist countries, students from East Asian collectivist countries, including Japan, reported the highest levels of social anxiety and acceptance of socially reticent behaviors (Schreier et al., 2010).

Collectivism and other traditional Japanese values appear to have a strong influence on the work culture in Japan. Social relationships are considered paramount in the workplace, where a cooperative attitude and self-sacrificing devotion to the team are highly valued qualities (Kawanashi, 2009, Kitanaka, 2012). As a result, employees tend to work long hours and be willing to take on large workloads. About $25 \%$ of Japanese companies reported their employees, on average, work more than 80 hours of overtime per month (Lane, 2017). While international surveys suggest Japan is not a leading country in average hours per worker, a large portion of overtime worked in Japan is believed to go unreported, and is often unpaid (Kawanashi, 2009). The willingness for employees to work significant overtime hours without pay, which is considered normal in Japanese work culture, is indicative of deeply rooted collectivist beliefs, in addition to traditionally esteemed values of loyalty, diligence, and hard work (Davies \& Ikeno, 2002). It also is generally frowned upon to leave work before your boss or to not participate in social activities with coworkers after work, which occur regularly (Lane, 2017). Overall, this work environment suggests that employees are likely to feel a high degree of pressure to be productive and competent, fit in, and maintain positive relationships with co-workers.
The perspectives and behaviors of Japanese workers seem to be influenced by long-term economic trends in addition to sociocultural factors. In the 1990s, Japan experienced a prolonged period of economic stagnation after an era, from the 1950 's to 1980 's, of growth and modernization. Failing businesses, wage cuts, and rising unemployment led to a shift in workforce sentiment, from that of job stability to financial insecurity and fear about the future. This shift was reinforced by the increasing use of temporary, or contracted, workers and a general move toward performance-based, rather than seniority-based, pay and promotion (Kawanishi, 2009). The sense of financial security and certainty about being employed at one company for life, which many Japanese workers enjoyed in the previous era, could no longer be justified in the 1990s and 2000s. In recent years, the Japanese economy has improved, however this uncertainty remains, adding another dimension of pressure for workers.

The perceived pressure of job insecurity may be especially high in Japan because of the stigma and shame associated with being fired, unemployed, or bankrupt in Japanese society. These misfortunes may be considered a loss of face and failure to meet the obligation to provide for one's family. Additionally, fired employees likely lose their friends and social network (Russell et al., 2016). While similar risks and consequences likely exist in other countries, their psychological impact appears particularly acute for Japanese people, given the fundamental importance of having a place within one's group.

Cultural and financial pressure, combined with long hours and little time off, puts Japanese workers at higher risk for high levels of stress and other mental health issues (Davies \& Ikeno, 2002). A survey by Japan's health ministry in 2006 showed $62 \%$ of workers reported high levels of stress and anxiety, citing human relationships, amount of work, content of work, and the future as key factors (Kawanishi, 2009). Employees also tend to not take vacation as a result of aforementioned cultural pressures, creating more risk for mental health issues to develop and less opportunity to be addressed. Employees are typically entitled 20 vacation days per year, but about $35 \%$ don't use any of those days (Lane, 2017). 


\section{Mental Illness \& Stigma}

Just as cultural values and economic factors have created significant risks for mental health issues in Japan's workforce, negative perceptions of mental illness and treatment has been a significant barrier to addressing those issues. Stigma against mental illness, while not unique to Japan, historically has been prevalent (Kawanashi, 2009). Stigmatizing attitudes have been influenced by Japan's history of mental health care policy, which emphasized long-term institutionalization (Ando, Yamaguchi, Aoki, \& Thornicroft, 2013, Kanata, 2016, Kanehara, Umeda, \& Kawakami, 2015). Modern mental health laws can be traced back to the Mental Hygiene Law passed in 1950, which established mental hospitals. Under that law, involuntary hospitalization was permitted with court approval or consent by a legal guardian. The Ministry of Health and Welfare also published a report in 1951 describing individuals with mental disorders as an economic risk factor, being that they do not engage in productive economic activity and prevent caretakers from doing so (Kanata, 2016). This stance propelled the institutionalization of the mentally ill, isolating them from the rest of society.

In the 1960s and 1970s, the government continued to promote psychiatric hospitalization in contrast to the global trend of deinstitutionalization, as mental illness was framed as a public safety issue. In 1984, the uncovering of severe mistreatment and suspicious deaths of numerous inpatients at one psychiatric hospital drew significant national and international attention, pressuring Japan's government to make changes to its mental health policies. In 1987, the National Mental Health Law was revised, emphasizing the protection of patient rights and supporting the establishment of social rehabilitation facilities, which was considered a first step toward deinstitutionalization. Since the 1990s, the Japanese government has stepped up efforts to improve the mental health care system and transition to community-based care. The number of inpatient psychiatric patients and their average length of stay has declined overtime, but Japan still has the highest proportion of psychiatric care beds per capita of any OECD country (Kanata, 2016).

The structural seclusion of people suffering from mental illness is reflected in stigmatic perceptions of mental illness and its treatment that exist in Japanese culture. A review of 19 studies of mental-health-related stigma in the Japanese general population showed that few people believed recovery from mental disorders was possible, and the majority endorsed keeping a greater social distance from those diagnosed (Ando et al., 2013). Additionally, almost two-thirds of individuals with mental illness never seek treatment from a health professional due to stigma (Ando et al., 2013). The World Mental Health Survey, conducted by the World Health Organization (WHO) from 2002 to 2006, revealed that the primary reason for not seeking mental health services in Japan was low perceived need, which was associated with negative perceptions of mental health treatments and their effectiveness (Kanehara et al., 2015).

The importance of fitting in and other core Japanese values, may, in part, account for the stigma against mental illness (Ando et al., 2013). Mental illness, particularly in work settings, may be viewed as an indication of defects in personality, such as laziness, immaturity, or lack of discipline (Kawanashi, 2009). In a survey of 2,000 Japanese adults, about $80 \%$ endorsed nervousness and weakness of character as causes of depression and schizophrenia, in addition to social factors (i.e. day-to-day problems, traumatic events and loss, problems from childhood) (Nakane 2005). On the other hand, recent efforts have been made to integrate mental health care into workplace and community settings. This indicates increasing awareness of the importance of such care and signs of a more accepting, or less stigmatizing attitude towards those who need it. In addition, attitudes towards mental illness in Japan, as well as cultural values, may not be as homogeneous as the literature suggests.

\section{Mental Health \& Prevention Measures}

In recent decades, mental health has become a prominent national issue in Japan, receiving increasing attention from the government and media. Of all mental health issues, suicide is perhaps the most salient in the Japanese media and mental health research. Japan has one of the highest rates of suicide in the world, although it has been declining since 2009 (Russell et al., 2016). According to a survey by the national health ministry conducted in 2017, 24\% of Japanese people had considered suicide, 19\% within the past year ("Nearly a quarter", 2017). Japan's work environment, particularly the high levels of overtime some employees work, has been implicated in some suicides and other deaths (Lane, 2017). Furthermore, being unemployed is a major risk factor for suicide in Japan, making up more than half of people who committed in 2008 (Kawanashi, 2009) and 60\% in 2013 (Russell et al., 2016). From 1985 to 2009 , suicide rates have been significantly correlated with unemployment rates (Yutaka, 2012). These figures suggest that financial pressures play a particularly important role in the mental health of Japanese people. As previously mentioned, being unemployed tends to bring about significant shame in Japanese culture and is closely tied to self-worth.

The decline in suicide rates since 2009 likely has resulted from preventative efforts by the Japanese government. In 2006, the government passed a bill to coordinate suicide-prevention efforts among government agencies, schools, and hospitals, set up suicide prevention centers, and provide public education (Kawanashi, 2009). In 2008, the program was further developed to focus on individuals with mental disorders, increase coordination between psychiatrists and medical doctors, and increase public knowledge about depression (Yutaka, 2012). In 2010, the national government led a campaign to raise awareness about suicide through TV commercials and other public outlets (Yutaka, 2012). The government has also recognized the financial and job-related risk factors for suicide and other mental health issues. In 2009, they expanded unemployment insurance and tightened regulations on temporary employment (Russell et al., 2016).

While suicide has received much attention, as well as targeted solutions from the government, there also have been efforts to improve the mental health care system as a whole, and mental health in the workplace. Repeated efforts have been made to transition from hospital-based to community-based care and provide a wider variety of services in that setting. In 2013, the Japanese government launched the "Regional Health Care Strategic Plan", designating mental disorders as the fifth "priority disease" for medical services, policy attention, and program development, along with cancer, stroke, heart attack, and diabetes (Ito, Frank, Nakatani, \& Fukuda, 2013). Under this plan, prefectural governments are required to expand 
mental health care services, access, and resources, and address prevention, recovery, and emergency care. While this initiative may lead to significant improvements to mental health care nationwide, several challenges have been identified. Many local governments have little or no experience developing mental health service plans, limited service capacity in local communities, and limits to available funding. Additionally, local governments may set minimum outcome targets to be perceived as successful, which was reported to occur in the implementation of plans for the other four priority diseases (Ito et al., 2013).

Recognizing the detriments of stress in the workplace, Japan has begun implementing reforms to identify and reduce it. In 2014, the Industrial Safety and Health Law was revised to require annual "stress checks" at companies with at least 50 employees. The new law mandates that companies provide employees a stress questionnaire and give them the option to consult a physician and receive information on stress management. Companies are also recommended to analyze stress survey responses, in aggregate, to improve the work environment (Kawakami \& Tsutsumi, 2016). The initiative has received criticism for focusing on individual assessment rather than improvements to work conditions, and not being evidenced-based. Multiple studies suggest the required aspect of the program (i.e. providing stress assessments and physician consultation) is not effective (Kawakami \& Tsutsumi, 2016), whereas the optional component (i.e. stress management education and work environment changes) is supported by empirical research (Yoshimura 2013). Additionally, critics argue that employees may be reluctant to participate and there is a lack of procedural guidelines for implementation (Kawakami \& Tsutsumi, 2016).

\section{Discussion: Advancing Workplace Mental Health by Targeting Risks \& Barriers}

The national initiatives to improve mental health care indicate commitment by the Japanese government and increasing awareness of the importance of mental health in Japanese society. While some progress has been made regarding suicide prevention and the system as a whole, reforms specific to Japan's work environment appear nascent. Based on research, theoretical perspectives, and historical mental health care initiatives in Japan, the primary impediments to mental health in the workplace fall under three categories: work culture, financial insecurity, and mental illness stigma. These are intersecting risks to mental health and barriers to treatment that together create a framework upon which mental health care initiatives can be based on and improved.

A work environment that promotes mental health and well-being should involve work-life balance, in which employees have a reasonable limit to how many hours they work and sufficient vacation. Furthermore, employers and managers ideally are accepting of, and even encourage, taking time off. The work culture in Japan, in which overtime and foregoing vacation are common, and it is frowned upon to leave work early or not socialize after, opposes the notion of work-life balance. Spurred by suicides and other deaths related to overwork, in addition to concerns about long-term economic growth, the Japanese government has sought to change this culture. In 2016, Prime Minister Shinzō Abe described "work style reform" as a key aspect of his policy agenda, seeking to improve work-life balance and reduce disparities in pay and working conditions between full-time and part-time employees, as well as males and females ("A prologue to workstyle reforms", 2018). The proposed reforms included a cap on overtime of 100 hours per month. Unfortunately, in 2018 Prime Minister Abe abandoned this proposal entirely after receiving criticism about faulty supporting data and facing a lack of consensus on many of the details ("Japan's Abe", 2018). Despite the lack of success of labor reforms thus far, work-life balance would likely improve should these changes occur. However, these reforms would be better framed as a public health initiative rather than part of economic policy.

Financial or job insecurity, identified as a second primary risk to mental health in the workplace, stems from recent economic instability and cultural fears of being fired or unemployed. The long-term economic outlook in Japan has been concerning given its aging population, which means an increasing proportion of the population will need to be supported, rather than contribute to economic productivity. These trends were a main reason why the Japanese government, led by Prime Minister Abe, has made significant modifications to economic policy over the past few years. While an aging population could have negative implications for the economic welfare of Japanese citizens, this also means that there is likely to be a systemic shortage of labor. Thus, job insecurity seems less likely to be an actual risk to Japanese workers. Still, there is likely to always be some level of economic cyclicality and uncertainty.

Regardless of economic conditions, the stress and fear associated with the risk of unemployment is likely to remain, given the cultural perspective of one's job as central to one's sense of self and worth. However, the potential detriments of becoming unemployed can be mitigated by supportive public services, which is precisely what Japan's government has attempted to improve upon. Since 2009, the government has expanded unemployment insurance, improved safety nets for economically disadvantaged families, and tightened regulations for temporary employment (Russell et al., 2016). These efforts will not completely remove the risks and worry associated with unemployment, but may create an environment, in addition to the aforementioned structural labor shortage, in which job insecurity only minimally contributes to the stress of Japanese workers.

Stigma against mental illness is a primary barrier to the development of mental health programs worldwide (Stuart, 2016). For the Japanese workforce, culturally-embedded stigma may discourage individuals from taking actions to address mental health issues, such as taking time off, working less hours, or seeking help. Furthermore, an environment absent of consideration or accommodation for mental illness allows for it to develop unnoticed and unimpeded, as seen in the numerous cases of suicides due to overwork. While some of the national mental health initiatives, such as those related to suicide prevention and workplace stress reduction, may have some positive impact on the public's perception of mental illness, initiatives directly targeting stigma are needed for significant change in perception to occur. Almost no such initiatives have existed in Japan on a national level. The renaming of schizophrenia from "mind-split disease" to "integration disorder" by the Japanese Society of Psychiatry and Neurology in 2002 was one exception, which has proven 
successful in reducing stigma towards people with psychosis over the long-term (Koike et al., 2015).

A national strategy to reduce stigma against mental illness in Japan can be informed by the extensive research available on numerous recent anti-stigma initiatives around the world, primarily in high-income countries. A meta-analysis of 79 programs from 14 countries found that programs centered around interpersonal contact, as opposed to education (i.e. literacy) or social activism (i.e. protest), were generally most effective for reducing stigma (Corrigan et al., 2012). These programs involve direct contact between people suffering from mental illness and the general public, or targeted groups. Education programs, such as mass media campaigns, have yielded mixed results and tend to be less cost-effective (Stuart, 2016). Opening Minds, an anti-stigma initiative which began in 2009 by the Mental Health Commission of Canada, is one program employing interpersonal contact that could be used as a model for Japan, given its documented success. Opening Minds created networks of community-based programs that deliver contact-based education to different target groups in Canada, including health care providers and students (Stuart, 2016). In Japan's case, anti-stigma initiatives that target employers may be an effective means to reduce stigma in the workplace.

\section{Conclusions}

Japan's collectivist, work-oriented culture and historically negative perceptions of mental illness are significant impediments to improving the mental health and well-being in their workforce. Japan's work culture, fearfulness of job loss, and stigma against mental illness are three distinct risks that can be targeted to improve mental health in the workplace. While Japan's government has implemented some reforms to address these risks, particularly regarding suicide prevention, initiatives specific to mental health in the workplace are in an early stage of planning and development. There is a need for more targeted investment in anti-stigma campaigns, further development of workplace stress-management programs, and renewed efforts to support work-life balance through legal reforms.

\section{References}

A Prologue to workstyle reforms. (2018) The Japan Times Ltd. Retrieved from https://www.japantimes.co.jp/opinion/2018/01/30/c ommentary/japan-commentary/prologue-workstyle-reforms/\#.Wr-i62inHrc.

Ando, S., Yamaguchi, S., Aoki, Y., Thornicroft, G. (2013) Review of mental-health-related stigma in Japan. Psychiatry and Clinical Neurosciences. 67(7). 471482.

Lane, E. (2017) The young Japanese working themselves to death. BBC News. Retrieved from http://www.bbc.com/news/business-39981997.

Japan's Abe abandons contentious labor reform after flawed data. (2018) CNBC LLC. Retrieved from https://www.cnbc.com/2018/02/28/japans-abeabandons-contentious-labour-reform-given-flaweddata.html.
Corrigan, P.W., Morris, S.B., Michaels, P.J, Rafacz, J.D., Rusch, N. (2012) Challenging the public stigma of mental illness; a meta-analysis of outcome studies. Psychiatric Services. 63(10). 963-973.

Davies, R.J., \& Ikeno, O. (2002) The Japanese mind: Understanding contemporary Japanese culture. Tuttle Publishing. North Clarendon, VT.

Ito, H., Frank, R.G., Nakatani, Y., Fukuda, Y. (2013) Mental health care reforms in Asia: the regional health care strategic plan: the growing impact of mental disorders in Japan. Psychiatric Services. 64(7). 617619.

Nearly a quarter of Japanese have considered suicide, government survey says (2017). The Japan Times Ltd. Retrieved from https://www.japantimes.co.jp/news/2017/03/21/nati onal/ nearly-quarter-japanese-considered-suicidegovernment-survey-says/\#.Wu3Wi9Mvyi4.

Kanata, T.K. (2016) Japanese mental health care in historical context: why did Japan become a country with so many psychiatric care beds? Social Work (Stellenbosch. Online). 52(4). 472-489.

Kanehara, A., Umeda, M., Kawakami, N. (2015) Barriers to mental health care in Japan: Results from the World Mental Health Japan Survey. Psychiatry and Clinical Neurosciences. 69(9). 523-533.

Kawakami, N., \& Tsutsumi, A. (2016) The Stress Check Program: a new national policy for monitoring and screening psychosocial stress in the workplace in Japan. Journal of Occupational Health. 58(1). 1-6.

Kawanishi, Y. (2009) Mental health challenges facing contemporary Japanese society: The 'lonely people'. Global Oriental. Kent, UK.

Kitanaka, J. (2012) Depression in Japan: Psychiatric cures for a society in distress. Princeton University Press.

Koike, S., Yamaguchi, S., Ojio, Y., Shimada, T., Watanabe, K., Ando, S. (2015). Long-term effect of a name change for schizophrenia on reducing stigma. Social Psychiatry and Psychiatric Epidemiology. 50(10). 1519-1526.

Russell, R., Metraux, D., Tohen, M. (2016) Cultural influences on suicide in Japan. Psychiatry and Clinical Neurosciences. 71(1). 2-5.

Schreier, S.S., Heinrichs, N., Alden, L., Rapee, R.M., Hofmann, S.G., Chen, J., Ja Oh, Kyung, Bogels, S. (2010) Social anxiety and social norms in individualistic and collectivistic countries. Depression and Anxiety. 27(12). 1128-1134. Stuart, H. (2016) Reducing the stigma of mental illness. Global Mental Health. 3. 17.

Yoshimura, K., Kawakami, N., Tsusumi, A., Inoue, A., Kobayashi, Y., Takeuchi, A., Fukudu, T. (2013) Cost-benefit analysis of primary prevention programs for mental health at the workplace in Japan. J-STAGE. Industrial Hygiene Journal. 55(1). $11-24$.

Yutaka, M. (2012) Suicide in Japan. The Lancet. 379(9823). 1282-1283. 